\title{
Modified Saponification and HPLC Methods for Analyzing Carotenoids from the Retina of Quail: Implications for Its Use as a Nonprimate Model Species
}

\author{
Matthew B. Toomey and Kevin J. McGraw
}

Purpose. To investigate carotenoid content in the retina of Japanese quail (Coturnix japonica), for comparison with carotenoids in human retina, and to assess the effects of different saponification procedures on the recovery of quail retinal carotenoids.

Methods. Extracted retinal carotenoids were saponified with methods adapted from recent studies, then identified and quantified with reverse-phase high-performance liquid chromatography (HPLC). To assess the effects of saponification conditions on carotenoid recovery from quail retina, we varied base concentration and the total time of saponification across a wide range and again used HPLC to compare carotenoid concentrations among conditions.

REsults. Astaxanthin and galloxanthin were the dominant carotenoids recovered in the quail retina, along with smaller amounts of five other carotenoids (lutein, zeaxanthin, 3 '-epilutein, $\varepsilon$-carotene, and an unidentified carotenoid). Astaxanthin was sensitive to saponification conditions; recovery was poor with strong bases $(0.2$ and $0.5 \mathrm{M} \mathrm{KOH})$ and best with weak bases $(0.01$ and $0.2 \mathrm{M} \mathrm{KOH})$. In contrast, xanthophyll carotenoids (galloxanthin, zeaxanthin, lutein, 3 '-epilutein, and the unknown) were best recovered with strong base after 6 hours of saponification at room temperature. The recovery of $\varepsilon$-carotene was not affected by saponification conditions.

Concuusions. Separate chemical hydrolysis procedures-using a strong base to recover xanthophylls and a weak base to recover astaxanthin-should be used for maximizing recovery of quail retinal carotenoids. Because the dominant carotenoids in quail retina are absent in human retina, and because of their different packaging (e.g., esterified in oil droplets) and lightabsorbance properties compared with xanthophylls in the human eye, use of the quail as a model organism for studying human retinal carotenoids should be approached with caution. (Invest Ophthalmol Vis Sci. 2007;48:3976-3982) DOI: 10.1167/iovs.07-0208

$\mathrm{X}$ anthophyll carotenoids, such as lutein and zeaxanthin, are thought to play a role in enhancing visual acuity, ${ }^{1-4}$ reducing photodamage, and preventing age-related macular degeneration ${ }^{5-7}$ in the human retina. In several recent studies, Japanese quail (Coturnix japonica) have been used as a model of human retinal carotenoid accumulation and function, be-

From the School of Life Sciences, Arizona State University, Tempe, Arizona.

Supported by Arizona State University, College of Liberal Arts and Sciences, School of Life Sciences, Tempe, Arizona.

Submitted for publication February 17, 2007; revised April 19 and May 16, 2007; accepted June 18, 2007

Disclosure: M.B. Toomey, None; K.J. McGraw, None

The publication costs of this article were defrayed in part by page charge payment. This article must therefore be marked "advertisement" in accordance with 18 U.S.C. $\$ 1734$ solely to indicate this fact.

Corresponding author: Matthew B. Toomey, School of Life Sciences, Arizona State University, Tempe, AZ 85287-4501;

matthew.toomey@asu.edu. cause the profile of quail retinal carotenoids appears to mimic that of humans ${ }^{8-11}$ and because quail retinal carotenoids are photoprotective. ${ }^{9,10}$ However, the retinal carotenoid profile reported in these recent high-performance liquid chromatography (HPLC)-based studies of quail ${ }^{8-11}$ differs from that in some of the first studies of quail retinal carotenoids. These early, thin-layer chromatography (TLC) and microspectrophotometry studies detected high quantities of a red, long-wavelength-absorbing ketocarotenoid-astaxanthin. ${ }^{12,13}$

In the avian retina, carotenoids are concentrated in lipidrich oil droplets and are typically esterified with fatty acids., ${ }^{814}$ These carotenoids must be hydrolyzed to remove esters before identification and quantification with conventional HPLC. The most commonly used method to hydrolyze carotenoid esters is base-catalyzed saponification, but astaxanthin is more sensitive to extraction conditions than are other retinal carotenoids (e.g., xanthophylls and carotenes), as a highly oxidized molecule that degrades under strong basic conditions ${ }^{15,16}$ and when using sonication. ${ }^{17}$ This raises the question of whether particular methods for carotenoid recovery explain why astaxanthin was not reported in recent HPLC studies of quail retina.

To investigate the presence of astaxanthin in the quail retina and the effects of chemical saponification on retinal carotenoid recovery, we conducted two separate HPLC-based experiments. In our first experiment, we compared saponification conditions derived from previous studies of quail ${ }^{8-11}$ to a method that has been used to recover esterified astaxanthin from an alga (Haematococcus pluvialis). ${ }^{16}$ We recovered astaxanthin with each of these methods, but we were unable to achieve optimal recovery of all retinal carotenoids with any one method. Therefore, we conducted a second experiment, in which we varied base concentration and the total time of saponification across a wide range, in an attempt to identify conditions that maximize the recovery of each type of carotenoid in the quail retina. The retinal carotenoid profile we observed differed from that in recent HPLC-based studies of quail, and we discuss the implications of this carotenoid profile for use of this species as a model of human retinal carotenoid accumulation and function.

\section{MethodS}

\section{Experiment 1}

Sample and Source Preparation. We acquired five adult male Japanese quail for study that were purchased from a local dealer (Pratt's Pet and Feed, Glendale, AZ) in January 2005. Since purchase, they had been housed by colleagues indoors under standard fluorescent lighting (12-hour light-dark cycle) and were fed Game Bird Maintenance Chow (Purina Mills, Inc., St. Louis, MO) and water ad libitum. From January to March 2005, our colleagues used these quail in noninvasive studies of thermoregulation that involved direct respirohygrometry and exposure to temperatures of $30^{\circ} \mathrm{C}$ to $32^{\circ} \mathrm{C}$ for up to 2 hours each day for no more than 4 days. ${ }^{18}$ The birds showed no adverse, long-term effects from this treatment. We assumed care of the birds in April 2005 and continued to feed them Maintenance Chow and water ad libitum for 7 months. At that time, we euthanatized the quail 
under a continuous stream of carbon dioxide, and the eyes were enucleated and refrigerated within 1 minute of death. Each eye was then hemisected under a dissecting scope, and the whole retina was removed and stored at $-80^{\circ} \mathrm{C}$ for 4 months. All procedures were approved by the Arizona State University Institutional Animal Care and Use Committee and were in accordance with the ARVO Statement for the Use of Animals in Ophthalmic and Vision Research.

To help understand the source and origins of carotenoids in quail retina, we measured carotenoid content of the diet ( $n=3$ replicates) according to the methods of McGraw et al. ${ }^{19}$ and found $0.38 \pm 0.06$ $\mu \mathrm{g} / \mathrm{g}$ of lutein, $0.41 \pm 0.07 \mu \mathrm{g} / \mathrm{g}$ of zeaxanthin, and less than $0.08 \mu \mathrm{g} / \mathrm{g}$ of both $\beta$-cryptoxanthin and $\beta$-carotene. We found no astaxanthin or galloxanthin in the quail diet.

Extraction of Carotenoids. The right and left retina from each individual was weighed to the nearest $10^{-5} \mathrm{~g}$ and ground together for 3 minutes at $30 \mathrm{~Hz}$ in a ball mill (MM200; Retsch GmbH \& Co. KG, Haan, Germany) in $2 \mathrm{~mL}$ of 1:1 hexane:tert-butyl methyl ether (MTBE). The ground retinas and solvent were transferred to a 9-mL culture tube and centrifuged for 5 minutes at $3000 \mathrm{rpm}$. The colored solvent fraction was then transferred to a 9-mL culture tube and evaporated to dryness under a stream of nitrogen. Carotenoids were resuspended in $6 \mathrm{~mL}$ of 1:1 hexane:MTBE, and $1 \mathrm{~mL}$ of this solution was transferred to each of six 9-mL culture tubes and evaporated to dryness under a stream of nitrogen, resulting in six identical samples from each individual. We also prepared and treated duplicate samples of pure astaxanthin standard (Sigma-Aldrich, St. Louis, MO) to examine the effects of saponification on a known concentration of free astaxanthin.

Saponification of Retinal Extracts. We used the saponification conditions (time, temperature, and base concentration) from recent studies ${ }^{8-11}$ of quail retinal carotenoids and compared these with the conditions suggested by Yuan and $\mathrm{Chen}^{16}$ for the complete hydrolysis of astaxanthin esters from $H$. pluvialis, with minimum degradation. In these studies, either sodium hydroxide $(\mathrm{NaOH})$ or potassium hydroxide $(\mathrm{KOH})$ was used in the saponification solutions. ${ }^{8-11,16}$ To address possible differential effects that these bases may have had on carotenoid degradation, we repeated each method with $\mathrm{KOH}$ and $\mathrm{NaOH}$. In method A, based on conditions used by Yuan and Chen, ${ }^{16}$ we prepared separate $0.02-\mathrm{M}$ solutions of $\mathrm{KOH}$ and $\mathrm{NaOH}$ in methanol and incubated astaxanthin standards as well as retinal carotenoids from each quail in $1 \mathrm{~mL}$ of each solution, under nitrogen for 6 hours in the dark at room temperature $\left(\sim 22^{\circ} \mathrm{C}\right)$. Method $\mathrm{B}$ was based on the conditions used by Khachik et al., ${ }^{8}$ who did not report obtaining astaxanthin in quail retina. Procedures were the same as in method A, with the following changes: $0.06-\mathrm{M}$ solutions of $\mathrm{KOH}$ and $\mathrm{NaOH}$ in methanol were used, and the samples were incubated as just described for 30 minutes. Method $\mathrm{C}$ was based on the conditions used by Thomson et al. ${ }^{9,10}$ and Toyoda et al., ${ }^{11}$ who also did not report astaxanthin in quail retina. Procedures were the same as those in method A, with the following changes: 1.32-M solutions of $\mathrm{KOH}$ and $\mathrm{NaOH}$ in methanol were used with $0.07 \mathrm{M}$ pyrogallol (an antioxidant) and were incubated as just described for 15 minutes.

After incubation, we added $1 \mathrm{~mL}$ of saturated sodium chloride in deionized water to each tube and shook the tubes vigorously by hand for $\sim 30$ seconds. We then added $2 \mathrm{~mL}$ of deionized water to each of the tubes and shook them. Finally, we added $3 \mathrm{~mL}$ of $1: 1$ hexane:MTBE to each of the tubes and shook them again. The tubes were centrifuged for 5 minutes at $3000 \mathrm{rpm}$, and the organic layer was transferred to a 9-mL culture tube and evaporated to dryness under a stream of nitrogen and prepared for HPLC analysis.

HPLC Analysis. We resuspended the saponified carotenoids in $200 \mu \mathrm{L}$ of a mobile phase that consisted of $44: 44: 12$ ( $\mathrm{vol} / \mathrm{vol} / \mathrm{vol}$ ) methanol:acetonitrile:dichloromethane, $50 \mu \mathrm{L}$ of which was injected into an HPLC system (Alliance 2695; Waters Corp., Milford, MA) fitted with a 5.0- $\mu \mathrm{m}$ column $(4.6 \times 250-\mathrm{mm}$; YMC Carotenoid; Waters $)$ and a built-in column heater set at $30^{\circ} \mathrm{C}$. We used a three-step gradient solvent system to analyze both xanthophylls and carotenes in a single run, at a constant flow rate of $1.2 \mathrm{~mL} / \mathrm{min}$; first, we used isocratic elution with 44:44:12 (vol/vol/vol) methanol:acetonitrile:dichloromethane for 11 minutes, followed by a linear gradient up to $42: 23: 35$ (vol/vol/vol) methanol:acetonitrile:dichloromethane for 21 minutes, and finishing with a return to the original isocratic conditions from 21 to 31 minutes. We identified and quantified carotenoids by comparing their retention times and absorbance spectra to those of external standards of purified zeaxanthin (DSM Inc., Heerlen, Netherlands), astaxanthin (Sigma-Aldrich), lutein (CaroteNature, Lupsingen, Switzerland), and $\beta$-carotene (CaroteNature). When external standards were not available, we tentatively identified carotenoids based on published absorbances (Table 1). External standards of galloxanthin were not available; therefore, in accordance with previous studies, ${ }^{23,24}$ we estimated galloxanthin concentration based on $\lambda_{\max }$ at $400 \mathrm{~nm}$ and on the calibration curve generated for our lutein standard $\left(\mathrm{E}_{1 \mathrm{~cm}}^{1 \%}=2550\right)$.

Statistical Analyses. All values are presented as the mean $\pm \mathrm{SE}$, and we report carotenoid recovery as nanograms carotenoid per whole retina. All comparisons are made within individual quail, as each of the samples extracted from a bird were expected to be the same. Statistical analyses were performed with commercial software (SPSS, ver. 13.0 for Windows; SPSS Inc. Chicago, IL). Data from experiment 1 met the assumptions of parametric statistics, and so we compared the recovery of specific carotenoids among saponification methods in a mixed-

TABLE 1. The Retention Times $\left(\mathrm{R}_{\mathrm{t}}\right)$, Absorbance Maxima $\left(\lambda_{\max }\right)$, Tentative Identification, and Concentration of Carotenoids in the Quail Retina

\begin{tabular}{|c|c|c|c|c|c|c|}
\hline Peak & $R_{t}(\min )$ & $\lambda_{\max }(\mathrm{nm})$ & & & Carotenoid & $\begin{array}{c}\text { Concentration } \\
\text { (ng/retina) }\end{array}$ \\
\hline 1 & 4.3 & & 401.9 & 422.5 & Galloxanthin*11,14,20,21 & $836.7 \pm 143.0$ \\
\hline 2 & 10.6 & & 370.6 & 475.8 & 13-cis-Astaxanthin $\dagger^{22}$ & $50.4 \pm 12.4$ \\
\hline 3 & 12.7 & & & 480.6 & Astaxanthin $\dagger^{22}$ & $336.5 \pm 89.3$ \\
\hline 4 & 13.7 & $(423.0)$ & 446.7 & 475.8 & $3^{\prime}$-Epilutein*8 & $168.5 \pm 24.1$ \\
\hline 5 & 14.8 & (421.3) & 446.7 & 474.6 & Lutein $\dagger$ & $51.3 \pm 6.8$ \\
\hline 6 & 17.5 & & 453.9 & 479.4 & Zeaxanthin $\dagger$ & $186.0 \pm 25.1$ \\
\hline 7 & 19.7 & $(422.5)$ & 447.9 & 477.0 & Unknown* & $42.2 \pm 7.3$ \\
\hline 8 & 21.9 & $(420.0)$ & 443.0 & 472.1 & $\varepsilon$-Carotene $\ddagger^{14}$ & $22.6 \pm 0.9$ \\
\hline
\end{tabular}

Concentration was determined based on the time and base combination in experiment 2 that provided the greatest recovery of each carotenoid. Data in parentheses denote the location of shoulders in the absorbance spectra. When an external standard was not available, carotenoids were identified by comparison with published absorbance spectra. Publications from which such data were taken are referenced by superscripts. Footnotes for symbols associated with carotenoid names denote the method by which each carotenoid was quantified. $\mathrm{R}_{\mathrm{t}}$, retention time; $\lambda_{\max }$, absorbance maximum.

* Quantified based upon the calibration curve for lutein standard.

$\dagger$ Identified and quantified by comparison to external standard.

‡ Quantified based on the calibration curve for $\beta$-carotene standard. 
model analysis of variance (ANOVA) with method (A, B, and C) and base $(\mathrm{KOH}$ and $\mathrm{NaOH})$ as fixed factors, individual as a random factor, and the interaction of base and method. Pair-wise comparisons between methods were made with the Tukey honest significant difference (HSD) post hoc test. Tests were considered statistically significant at the level of $P<0.05$.

\section{Experiment 2}

None of the methods used in experiment 1 optimally recovered all the carotenoids found in the quail retina (see the Results section). To address the limitations of experiment 1 and further evaluate saponification conditions for maximum recovery of quail retinal carotenoids, we conducted a second experiment. In this experiment, we manipulated the saponification conditions (time, temperature, and base concentration) across a wide range and assessed carotenoid recovery from quail retinas with HPLC.

Sample and Source Preparation. In August 2006, two adult male and two adult female Japanese quail were purchased from the same source as were the birds used in experiment 1 , retinal tissue was immediately collected, and carotenoids were extracted as described earlier. The quail dealer acquired their birds from a variety of sources and thus was unable to provide information about the history of diet and light environment of these individuals. However, we found that the plasma carotenoid types and concentrations of these quail were consistent with those in previous studies. ${ }^{8,11}$ Plasma carotenoids were dominated by lutein $(1.04 \pm 0.24 \mu \mathrm{g} / \mathrm{mL})$ and zeaxanthin $(0.26 \pm 0.04$ $\mu \mathrm{g} / \mathrm{mL})$, and we found no evidence of astaxanthin or galloxanthin in the plasma.

Saponification of Retinal Extracts. Pooled retinal extracts from each individual were separated into five identical samples in 9-mL culture tubes and evaporated to dryness under a stream of nitrogen. We then prepared five separate solutions of $0.01,0.02,0.1,0.2$, and 0.5 $\mathrm{M} \mathrm{KOH}$ in methanol and added $2 \mathrm{~mL}$ of one of these solutions to each sample of retinal carotenoids. The samples were incubated under nitrogen at room temperature $\left(\sim 22^{\circ} \mathrm{C}\right)$ in darkness. At 1, 2, 4, 6, and
8 hours, $350-\mu \mathrm{L}$ aliquots were removed from each sample and carotenoids were extracted as described in experiment 1.

HPLC Analysis. HPLC analysis was performed as described in experiment 1 , with the following changes. To improve peak resolution and reduce tailing of the astaxanthin peaks, the column was conditioned with orthophosphoric acid $\left(\mathrm{H}_{3} \mathrm{PO}_{4}\right) .^{25}$ Before carotenoid analysis, a $1 \%$ solution of $\mathrm{H}_{3} \mathrm{PO}_{4}$ in methanol was pumped through the column at $1.2 \mathrm{~mL} / \mathrm{min}$ for 40 minutes. Like previous empiric studies in which columns were prewashed with acid, ${ }^{26,27}$ we found no effect of this acid treatment on carotenoid degradation or recoveries (Toomey MB, unpublished data, 2007). We then used a revised three-step gradient solvent system to ensure the separation of the astaxanthin and lutein peaks. At a constant flow rate of $1.2 \mathrm{~mL} / \mathrm{min}$, we first used isocratic elution with $48: 48: 4$ ( $\mathrm{vol} / \mathrm{vol} / \mathrm{vol})$ methanol:acetonitrile:dichloromethane for 11 minutes, followed by a linear gradient up to 42:23:35 ( $\mathrm{vol} / \mathrm{vol} / \mathrm{vol})$ methanol:acetonitrile:dichloromethane for 25 minutes, and finishing with a return to the original isocratic conditions from 25 to 40 minutes.

Statistical Analyses. We compared the effects of base concentration and time on the recovery of individual retinal carotenoids with repeated-measures ANOVA with time and the interaction of base concentration and time as the within-subject factors and base concentration as the between-subject factor. Pair-wise comparisons between times and base concentrations were made with the Tukey HSD post hoc test.

\section{Results}

\section{Experiment 1}

Unlike previous HPLC studies, ${ }^{8-11}$ we recovered astaxanthin with each saponification method (A, B, and C) from quail retina (Fig. 1). Using these methods, we also detected three other carotenoids: galloxanthin, zeaxanthin, and $\varepsilon$-carotene. One carotenoid remained unidentified. This pigment was difficult to

a.

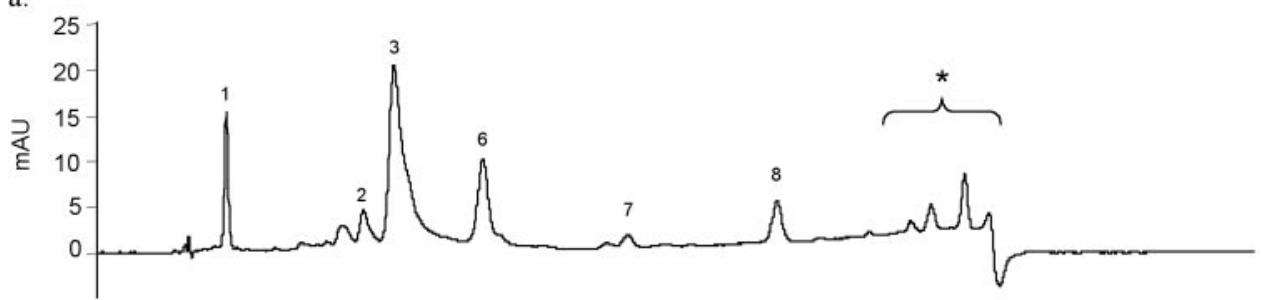

b.

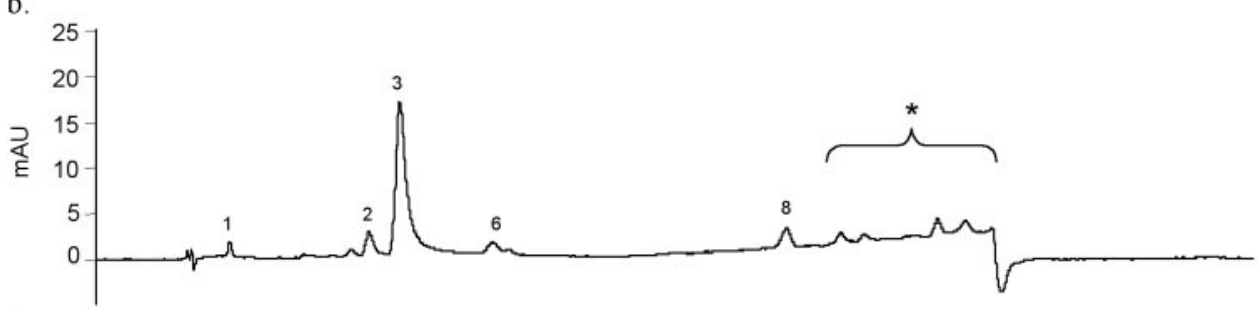

c.

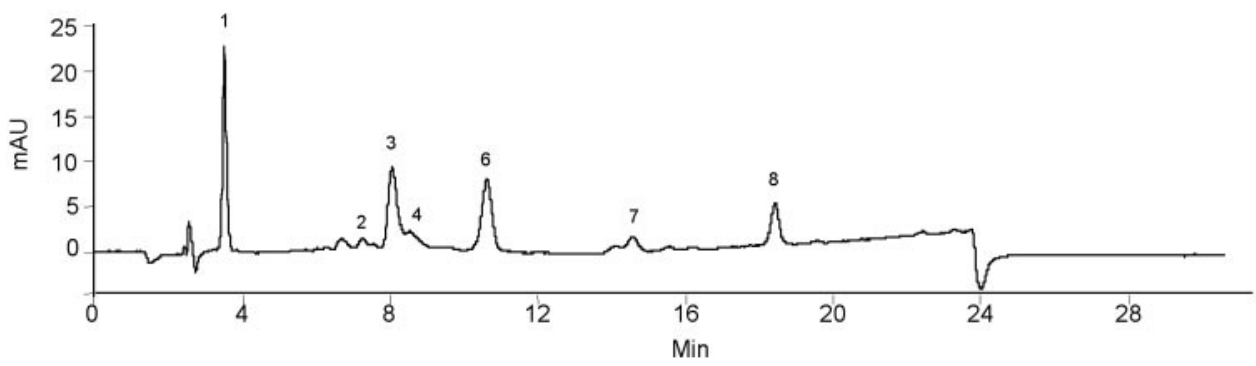

Figure 1. Two-dimensional HPLC chromatograms of the carotenoid profile of retinal extracts from one quail that were saponified with methods A (a), B (b), and C (c) in experiment 1 . Numbered peaks are identified in Table 1. Asterisks next to lateeluting peaks represent esterified forms of the identified carotenoids. Note in (c) that peaks 3 and 4 partially coeluted. 
TABLE 2. Retinal Carotenoid Recovery with Three Saponification Methods Adapted from Published Studies

\begin{tabular}{|c|c|c|c|c|c|c|c|}
\hline Method & Base & Galloxanthin* & Astaxanthin* & Zeaxanthin* $†$ & Unknown $\ddagger$ & $\varepsilon$-Carotene* & Astaxanthin Standard (ng) \\
\hline \multirow[t]{2}{*}{ A } & $\mathrm{KOH}$ & $543.0 \pm 64.6$ & $654.2 \pm 79.7$ & $352.8 \pm 40.0$ & $52.7 \pm 5.8$ & $15.1 \pm 0.1$ & $111.7 \pm 26.2$ \\
\hline & $\mathrm{NaOH}$ & $516.2 \pm 61.6$ & $619.6 \pm 101.6$ & $313.4 \pm 30.1$ & $53.7 \pm 6.7$ & $15.2 \pm 0.1$ & $63.0 \pm 18.4$ \\
\hline \multirow[t]{2}{*}{$\mathrm{B}$} & $\mathrm{KOH}$ & $153.9 \pm 17.4$ & $507.3 \pm 39.2$ & $90.2 \pm 3.7$ & 0.00 & $14.9 \pm 0.2$ & $56.0 \pm 40.5$ \\
\hline & $\mathrm{NaOH}$ & $164.6 \pm 15.6$ & $526.8 \pm 38.8$ & $84.9 \pm 5.8$ & $5.7 \pm 5.7$ & $14.8 \pm 0.1$ & $56.2 \pm 29.5$ \\
\hline \multirow[t]{2}{*}{ C } & $\mathrm{KOH}$ & $820.1 \pm 89.4$ & $198.7 \pm 20.2$ & $281.5 \pm 29.4$ & $57.4 \pm 5.3$ & $14.9 \pm 0.2$ & $32.7 \pm 18.8$ \\
\hline & $\mathrm{NaOH}$ & $693.2 \pm 65.0$ & $128.2 \pm 15.3$ & $154.0 \pm 14.1$ & $37.7 \pm 2.4$ & $14.6 \pm 0.1$ & $13.2 \pm 9.2$ \\
\hline
\end{tabular}

Data are the mean nanograms per retina \pm SE, unless otherwise noted. Methods A, B, and C were adapted from Yuan and Chen ${ }^{16}$, Khachik et al., ${ }^{8}$ and Toyoda et al. ${ }^{9}$, respectively.

* Carotenoid recovery differed significantly between all methods (Tukey HSD, $P \leq 0.049$ ).

† Carotenoid recovery differed significantly between bases $\left(\mathrm{F}_{1,20}=12.67, P=0.012\right)$. These differences may be an artifact of solution preparation.

‡ Carotenoid recovery differed significantly between methods A and B, and, B and C (Tukey HSD, $P \leq 0.001$ ).

characterize because it was present in very small amounts and because its retention time and absorbance spectrum did not match those of putative pigments eluting around that time (e.g., anhydrolutein, $\beta$-cryptoxanthin).

The saponification methods differed significantly in carotenoid recovery for each carotenoid measured (galloxanthin: $\mathrm{F}_{2,22}=139.6, P<0.001$, astaxanthin: $\mathrm{F}_{2,22}=53.4, P<0.001$, unknown: $\mathrm{F}_{2,22}=116.7, P<0.001$, zeaxanthin: $\mathrm{F}_{2,22}=77.4$, $P<0.001, \varepsilon$-carotene: $\left.\mathrm{F}_{2,22}=6.3, P=0.007\right)$. With method $\mathrm{A}$, we recovered significantly more astaxanthin, zeaxanthin, and $\varepsilon$-carotene than with methods $\mathrm{B}$ and $\mathrm{C}$; and with method $\mathrm{C}$, we recovered significantly more galloxanthin than with methods $\mathrm{A}$ and $\mathrm{B}$ (Table 2). With methods $\mathrm{A}$ and $\mathrm{C}$, we recovered significantly more of the unknown carotenoid than with method $\mathrm{B}$ (Table 2). The recovery of the purified astaxanthin standard was similar to retinal astaxanthin, with the highest amounts recovered using method A (Table 2). Saponification with $\mathrm{KOH}$ resulted in significantly more recovered zeaxanthin than did saponification with $\mathrm{NaOH}$ (Table 2), and the interaction of base type and method significantly affected the recovery of zeaxanthin $\left(\mathrm{F}_{2,20}=5.1, P=0.02\right)$ and the unknown carotenoid $\left(\mathrm{F}_{2,20} \stackrel{2,20}{=} 7.0, P=0.005\right)$. The effect of base on the recovery of zeaxanthin and the unknown carotenoid appears to be greater in method $\mathrm{C}$ than other methods (Table 2). These results are surprising, because we expected equivalent molar amounts of these bases to be equally effective. These differences may be an artifact of solution preparation, because $\mathrm{NaOH}$ is more deliquescent and less soluble in methanol than is $\mathrm{KOH}$, which may have led to lower concentrations of $\mathrm{NaOH}$ in solution than expected.

None of the three methods adapted from the literature resulted in optimal recovery of all the carotenoids in the quail retina. Esterified carotenoids were still present in samples treated with methods A and B (Figs. 1a, 1b), and these methods yielded relatively low recoveries of galloxanthin and the unknown carotenoid (method B). We did not observe esterified carotenoids in samples treated with method C (Fig. 1c); however, the amount of astaxanthin recovered with this method was significantly lower than that recovered with the other methods (Table 2). Also, in this experiment we did not observe 3 '-epilutein or lutein, which are known to be important carotenoids in the quail retina, because these carotenoids partially coeluted with astaxanthin (Fig. 1c). The astaxanthin peak also exhibited tailing and eluted over a 2 -minute period (Fig. 1). These analytical limitations were alleviated in experiment 2.

\section{Experiment 2}

Treatment of the column with $\mathrm{H}_{3} \mathrm{PO}_{4}$ reduced tailing and produced a symmetrical astaxanthin peak that eluted over $\sim 0.8$ minute (compare peak 3 in Fig. 1a to that in Fig. 2). Our revised HPLC method (mobile phase, 48:48:4) provided us with baseline resolution of astaxanthin, $3^{\prime}$-epilutein, and lutein, whose peaks eluted $\geq 1$ minute apart (Fig. 2).

The effects of base concentration and time on retinal carotenoid recovery were specific to the carotenoid type measured. Galloxanthin recovery differed significantly with base concentration and time (Table 3, Fig. 3). Strong bases (0.5 and $0.2 \mathrm{M}$ $\mathrm{KOH})$ recovered significantly more galloxanthin than the weak bases $(0.01$ and $0.02 \mathrm{M} \mathrm{KOH})$ that we tested (Tukey HSD, $P<$ 0.055 , Fig. 3). Galloxanthin recovery increased with the length of saponification; however, recovery appeared to plateau after 6 hours, and there was no significant difference in the recovery at 6 and 8 hours (Tukey HSD, $P>0.5$, Fig. 3). Astaxanthin recovery differed significantly among base concentrations (Table 3), with weak bases ( 0.01 and $0.02 \mathrm{M} \mathrm{KOH}$ ) affording recovery of more astaxanthin than did the stronger bases.
Figure 2. A representative two-dimensional HPLC chromatogram of the retinal carotenoid profile from one quail used in experiment 2 . The extract was saponified with $0.1 \mathrm{M}$ KOH for 6 hours. Numbered peaks are identified in Table 1.

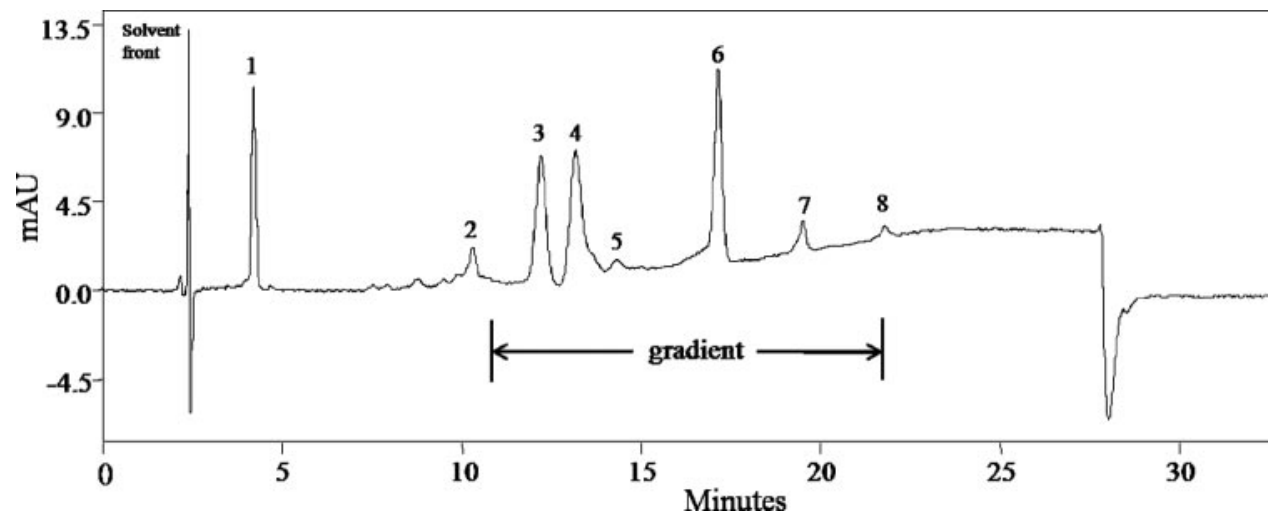


TABLE 3. Repeated-Measures ANOVA Table Showing the Effects of Time, Base Concentration, and Their Interaction on the Recovery of Quail Retinal Carotenoids in Experiment 2

\begin{tabular}{|c|c|c|c|c|c|c|c|c|c|c|c|c|c|c|c|}
\hline & \multirow[b]{2}{*}{$d f$} & \multicolumn{2}{|c|}{ Galloxanthin } & \multicolumn{2}{|c|}{ Astaxanthin } & \multicolumn{2}{|c|}{$3^{\prime}$-Epilutein } & \multicolumn{2}{|c|}{ Lutein } & \multicolumn{2}{|c|}{ Zeaxanthin } & \multicolumn{2}{|c|}{ Unknown } & \multicolumn{2}{|c|}{$\begin{array}{c}\varepsilon- \\
\text { Carotene }\end{array}$} \\
\hline & & $\mathbf{F}$ & $\boldsymbol{P}$ & $\mathbf{F}$ & $\boldsymbol{P}$ & $\mathbf{F}$ & $\boldsymbol{P}$ & $\mathbf{F}$ & $\boldsymbol{P}$ & $\mathbf{F}$ & $\boldsymbol{P}$ & $\mathbf{F}$ & $\boldsymbol{P}$ & $\mathbf{F}$ & $\boldsymbol{P}$ \\
\hline Base & 4,15 & 6.7 & 0.003 & 3.4 & 0.04 & 33.1 & $<0.001$ & 24.3 & $<0.001$ & 15.7 & $<0.001$ & 9.5 & $<0.001$ & 0.4 & 0.8 \\
\hline Time & 4,60 & 35.1 & $<0.001$ & 0.8 & 0.44 & 7.0 & 0.005 & 18.04 & $<0.001$ & 24.7 & $<0.001$ & 2.3 & 0.1 & 2.5 & 0.08 \\
\hline Time $\times$ base & 16,60 & 1.2 & 0.3 & 6.5 & $<0.001$ & 1.0 & 0.5 & 3.4 & 0.001 & 2.4 & 0.03 & 1.2 & 0.3 & 0.6 & 0.8 \\
\hline
\end{tabular}

The probabilities of the within-subject factors (time and time $\times$ base) are adjusted with Greenhouse-Geisser correction, to account for violations of sphericity. Statistically significant results are denoted in bold.

There was a significant effect of the interaction between base concentration and saponification time on astaxanthin recovery (Table 3). Astaxanthin recovery increased with time with the weak bases, but declined over time in samples treated with strong bases (Fig. 3). Zeaxanthin recovery differed significantly with base concentration, time, and the interaction of base concentration and time (Table 3, Fig. 3). Significantly more zeaxanthin was recovered with strong bases $(0.5,0.2,0.1 \mathrm{M}$ $\mathrm{KOH})$ than with weak bases $(0.01$ and $0.02 \mathrm{M} \mathrm{KOH}$; Tukey HSD, $P<0.003$; Fig. 3). Zeaxanthin recovery increased initially with duration of saponification, especially with strong bases, but plateaued after 6 hours, and there were no significant differences in recovery between 6 and 8 hours (Tukey HSD, $P>0.5$; Fig. 3). The recovery of 3 '-epilutein differed significantly with base concentration and time (Table 3, Fig. 3). Significantly more 3 '-epilutein was recovered with strong bases $(0.5$ and $0.2 \mathrm{M} \mathrm{KOH})$ than with the weak bases $(0.01$ and 0.02 $\mathrm{M} \mathrm{KOH}$; Tukey HSD, $P<0.027$, Fig. 3), and recovery increased with the length of saponification (Fig. 3). 3'-Epilutein recovery plateaued after 4 hours of saponification, and there were no significant differences in recovery between the 4-, 6-, and 8-hour periods (Tukey HSD, $P>0.5$, Fig. 3). Lutein recovery differed significantly with base concentration, time, and the interaction of base concentration and time (Table 3, Fig. 3). With strong bases $(0.5,0.2$, and $0.1 \mathrm{M} \mathrm{KOH})$ significantly more lutein was recovered than with weak bases $(0.01$ and $0.02 \mathrm{M}$ $\mathrm{KOH}$; Tukey HSD, $P<0.001$, Fig. 3). Lutein recovery also increased initially with the length of saponification, especially for strong bases, but plateaued after 4 hours, and there were no significant differences in recovery between 4,6 , and 8 hours (Tukey HSD, $P>0.3$, Fig. 3). Recovery of the unknown carotenoid differed significantly with base concentration but was not affected by the length of saponification (Table 3, Fig. $3)$. The strongest base treatments $(0.5$ and $0.2 \mathrm{M} \mathrm{KOH})$ resulted in recovery of significantly more of the unknown carotenoid than did the weakest bases $(0.01$ and $0.02 \mathrm{M} \mathrm{KOH}$; Tukey HSD,
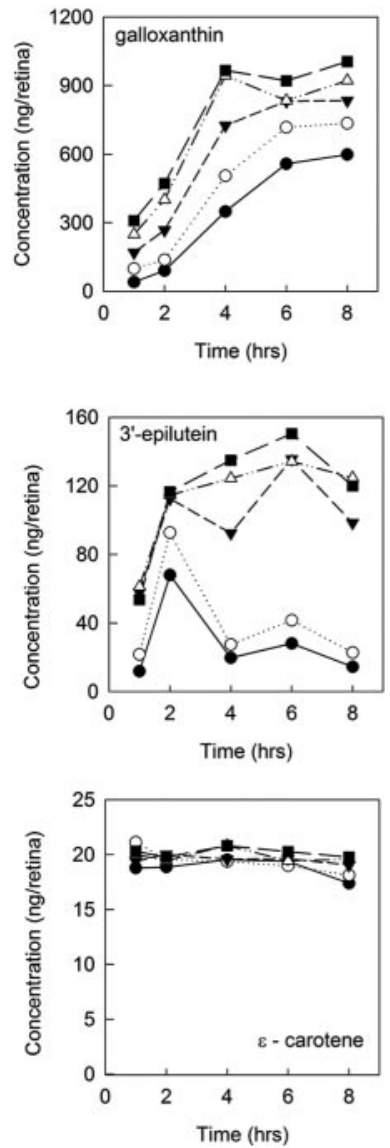
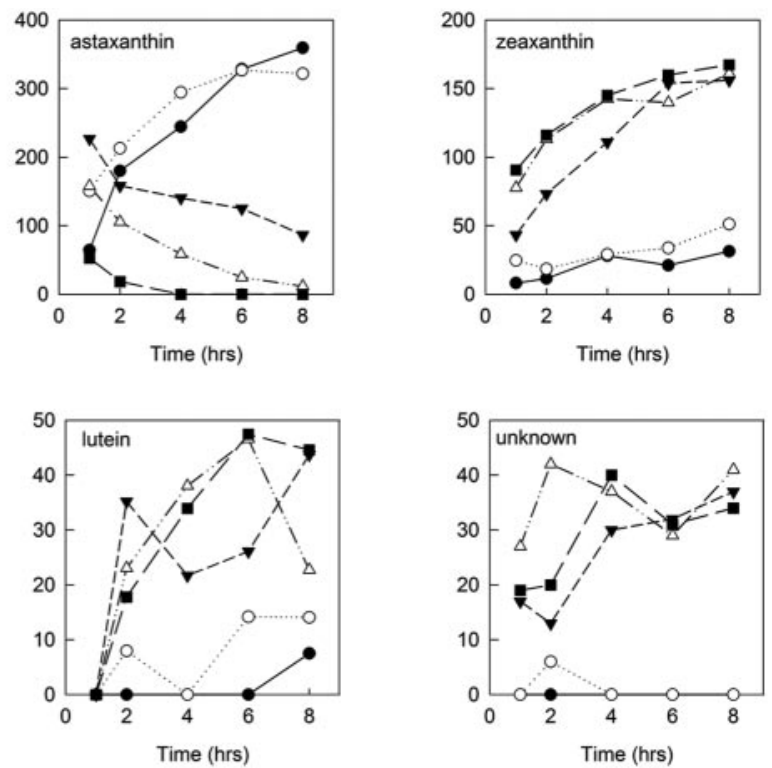

FIGURE 3. Mean recovery over time of quail retinal carotenoids during saponification with varying concentrations of $\mathrm{KOH}$ in methanol $(n=4)$, as performed in experiment 2 . 
$P<0.05$, Fig. 3). Base concentration and the length of saponification had no significant effect on the recovery of $\varepsilon$-carotene (Table 3).

\section{Relative Amounts of Different Carotenoids in the Quail Retina}

The retinal carotenoid profile observed in our study differed substantially from those reported in previous HPLC studies of the quail retina. ${ }^{8,11}$ Galloxanthin was the most concentrated quail retinal carotenoid in our study, and we found it in much higher concentrations than previously reported for any carotenoid found in the quail retina (Table 1). ${ }^{8,11}$ Astaxanthin was also highly concentrated in the retina; followed by zeaxanthin and 3 '-epilutein; and lesser amounts of lutein, $\varepsilon$-carotene, and an unknown carotenoid (Table 1). Zeaxanthin and lutein have recently been reported to be the dominant carotenoids in the quail retina, ${ }^{8-11}$ making up $>70 \%$ of the total in birds receiving a standard diet; however, our results indicate that these carotenoids make up less than $25 \%$ of the total. In our study, we found that lutein (lutein and 3 '-epilutein together) and zeaxanthin occur in relatively equal proportions in the quail retina $(219.8 \pm 30.9$ and $186.0 \pm 25.1 \mathrm{ng}$, respectively). Previous studies have indicated that lutein and zeaxanthin occur in a 1:2 ratio, similar to the distribution in the human retina. ${ }^{8-11}$ It is not clear whether the differences among studies are the result of extraction methods or the nutritional history of the quail. The quail in experiment 2 were collected directly from the supplier and their nutritional history was not known; note that the quail in experiment 1 with a known nutritional history had much higher retinal zeaxanthin levels than those in experiment 2 (compare Tables 1 and 2).

\section{Discussion}

We compared and modified published saponification and HPLC methods ${ }^{8-11}$ to investigate the presence of astaxanthin in the retina of Japanese quail and to examine the effects of different chemical hydrolysis conditions on the recovery of quail retinal carotenoids. Corroborating findings from early TLC and microspectrophotometry research, ${ }^{12,13}$ we found that astaxanthin was a dominant carotenoid in quail retina (the second most concentrated behind galloxanthin in our study). Mild saponification conditions, adapted from Yuan and Chen, ${ }^{16}$ yielded greater recoveries of astaxanthin than did either of the methods adapted from prior quail studies, ${ }^{8-11}$ in which stronger bases were used. We recovered astaxanthin with all saponification conditions that we tested, including those derived from studies that did not report astaxanthin from quail eyes. ${ }^{8-11}$ This result suggests that, although astaxanthin clearly degrades in strong alkali solutions, saponification conditions alone fail to explain why astaxanthin was not reported in prior work.

We see two potential explanations for why our study, but no prior HPLC study, detected the presence of astaxanthin in quail retina. First, we (and investigators in other studies ${ }^{8}$ ) had limited information about the rearing and dietary conditions of the farmed quail purchased for study, and so differences in quail nutritional history across studies may have contributed to retinal carotenoid differences. Second, we did not exactly replicate the extraction and HPLC conditions of these previous studies (rather, we targeted key parameters identified in the available literature), so we cannot be sure whether factors such as sonication during extraction or HPLC system conditions affected their recovery of astaxanthin. On this note, we observed that tailing of the astaxanthin peak can increase with column use/age and can become so extreme ( $>10$ minutes) that astaxanthin, but no other retinal carotenoids, becomes undetectable. Such tailing can be avoided by acidifying the stationary phase of the column, as we did in experiment 2 and has been done in prior work on astaxanthin in salmon (Salmo salar) and humans. ${ }^{25-27}$ In the end, however, we predict that astaxanthin is ubiquitous in the retinas of diurnal birds, based on its presence in other granivorous species, both domesticated and wild, that do not consume astaxanthin (e.g., zebra finch, house finch; Toomey MB, unpublished data, 2007), and based on the fact that red (R-type) oil droplets with an astaxanthin-typical absorbance spectrum have been observed in all diurnal birds investigated to date. ${ }^{14,28}$

We found (in experiment 2) that no single saponification condition maximized the recovery of all the carotenoids in quail retina. Given the different molecular characteristics of the range of carotenoids recovered, this finding is not surprising. Astaxanthin is comparatively more oxidized than the other retinal carotenoids, making it more susceptible to degradation; hence, it was maximally recovered with weak bases $(0.01$ and $0.02 \mathrm{M}$ ) after 6 hours of saponification. Other less oxidized xanthophylls, including 3 '-epilutein, zeaxanthin, and galloxanthin, were maximally recovered after 6 hours of saponification with strong base $(0.1-0.5 \mathrm{M})$. Thus, to maximize the recovery and quantification of the full complement of avian retinal carotenoids with alkali saponification, we suggest dividing extracts of each sample in two, and saponifying one portion with weak base to hydrolyze astaxanthin esters and the other portion with a strong base to hydrolyze xanthophyll esters. Because recoveries of each carotenoid plateaued after 4 to 6 hours and there were no significant differences between samples saponified for 6 or 8 hours, our results indicate that 6 hours is a sufficient time for saponification of quail retinal carotenoids using either method (at room temperature and in the absence of light and oxygen). Future investigations should consider enzymatic hydrolysis ${ }^{29}$ as a single method that can simultaneously recover esterified retinal xanthophylls and astaxanthin. However, enzymatic hydrolysis is also likely to vary in effectiveness, depending on the type of carotenoid ester being treated, ${ }^{30}$ and require a similar large-scale experimental approach to evaluate its potential for use with avian retinal carotenoids.

\section{Implications for Studies of Avian and Human Visual Function and Health}

The quail, ${ }^{8,11}$ and recently the chicken (Gallus gallus domesticus), ${ }^{31}$ have been proposed as model species for the study of retinal carotenoids in humans because of the reported similarities in the types, amounts, and functions of retinal carotenoids in each. Our results suggest that the retinal carotenoid profile of quail is substantially different from that of humans. Lutein, 3 '-epilutein, and zeaxanthin are the dominant carotenoids in the human retina, constituting greater than $85 \%$ of total carotenoids, ${ }^{8,32}$ but in our study of quail retina, these carotenoids comprised less than $24 \%$. Rather, the quail retina is dominated by galloxanthin and astaxanthin, both of which are not found in the human retina. 8,32 Chicken retina also contains these two carotenoids. ${ }^{23,33-35}$

This difference in the carotenoid profile, in addition to several other attributes of avian retinal carotenoids, forces us to re-evaluate whether quail (and birds generally) represent ideal models for the study of human retinal carotenoids. We recommend that future studies test the functional importance of the following three differences between avian and human retinal carotenoids before birds become preferred models for human research in this area. First, galloxanthin and astaxanthin in the avian eye absorb a wider range of light wavelengths $\left(\lambda_{\max }=\right.$ $403-480 \mathrm{~nm}$ ) than the xanthophylls reported from the human retina. 8,32 This characteristic in turn may offer broader cone photoprotection to birds than to humans. Second, carotenoids 
in the avian retina are "packaged" differently, esterified and in oil droplets, ${ }^{8,14}$ than are the membrane-bound, unesterified carotenoids associated with human retinal cones, ${ }^{32}$ and this packaging may enhance carotenoid stability/longevity and thus the duration of photoprotection in birds compared with humans. Finally, the fact that avian oil-droplet carotenoids are thought to be coupled to specific cone types (e.g., astaxanthin to long-wavelength-sensitive cones, lutein/zeaxanthin to medium-wavelength-sensitive cones, galloxanthin to short-wavesensitive cones, based on microspectrophotometric evidence $^{14}$ ) may have implications for the specificity of cone photoprotection by particular carotenoids. Manipulations of one dietary carotenoid (zeaxanthin, for example) may be predicted to increase photoprotection for only the type of cone in which it is housed, unless it serves as the precursor for the formation of (and increases concentrations of) metabolites, such as astaxanthin or galloxanthin, in other cone types. Much more work is needed to understand product-precursor relationships among avian retinal carotenoids so that we can determine how few or many photoreceptor classes gain protection by particular dietary or metabolically derived carotenoids.

In the end, our study by no means invalidates previous findings that retinal carotenoids in quail are diet-dependent and enhance photoprotection. ${ }^{9,10}$ Instead, we present improved techniques for recovering and analyzing avian retinal carotenoids and suggest important considerations for the use of quail and other avian species as models for studying carotenoid pigments in human retinas.

\section{Acknowledgments}

The authors thank Glenn Walsberg and Ty Hoffman for providing some of the quail used in this study and Bobby Fokidis, Lisa A. Taylor, and three anonymous reviewers for providing valuable comments on the manuscript.

\section{References}

1. Hammond BR, Wooten BR, Curran-Celentano J. Carotenoids in the retina and lens: possible acute and chronic effects on human visual performance. Arch Biochem Biophys. 2001;385:41-46.

2. Kvansakul J, Rodriguez-Carmona M, Edgar DF, et al. Supplementation with the carotenoids lutein or zeaxanthin improves human visual performance. Ophthalmic Physiol Opt. 2006;26:362-371.

3. Rodriguez-Carmona M, Kvansakul J, Harlow JA, Köpcke W, Schalch W, Barbur JL. The effects of supplementation with lutein and/or zeaxanthin on human macular pigment density and colour vision. Opbthalmic Pbysiol Opt. 2006;26:137-147.

4. Wooten BR, Hammond BR. Macular pigment: influences on visual acuity and visibility. Prog Retin Eye Res. 2002;21:225-240.

5. Ahmed SS, Lott MG, Marcus DM. The macular xanthophylls. Surv Ophthalmol. 2005;50:183-193.

6. Krinsky NI, Landrum JT, Bone RA. Biologic mechanisms of the protective role of lutein and zeaxanthin in the eye. Annu Rev Nutr. 2003;23:171-201.

7. Chiu C, Taylor A. Nutritional antioxidants and age-related cataract and maculopathy. Exp Eye Res. 2007;84:229-245.

8. Khachik F, de Moura FF, Zhao DY, Aebischer CP, Bernstein PS. Transformations of selected carotenoids in plasma, liver, and ocular tissues of humans and in nonprimate animal models. Invest Ophthalmol Vis Sci. 2002;43:3383-3392.

9. Thomson LR, Toyoda Y, Langner A, et al. Elevated retinal zeaxanthin and prevention of light-induced photoreceptor cell death in quail. Invest Ophthalmol Vis Sci. 2002;43:3538-3549.

10. Thomson LR, Toyoda Y, Delori FC, et al. Long term dietary supplementation with zeaxanthin reduces photoreceptor death in light-damaged Japanese quail. Exp Eye Res. 2002;75:529-542.

11. Toyoda Y, Thomson LR, Langner A, et al. Effect of dietary zeaxanthin on tissue distribution of zeaxanthin and lutein in quail. Invest Ophthalmol Vis Sci. 2002;43:1210-1221.
12. Meyer DB, Stuckey SR, Hudson RA. Oil droplet carotenoids of avian cones. 1. Dietary exclusion-models for biochemical and physiological studies. Comp Biochem Physiol. 1971;40:61-70.

13. Bowmaker JK, Kovach JK, Whitmore AV, Loew ER. Visual pigments and oil droplets in genetically manipulated and carotenoid deprived quail: a microspectrophotometric study. Vision Res. 1993;33:571-578.

14. Goldsmith TH, Collins JS, Licht S. The cone oil droplets of avian retinas. Vision Res. 1984;24:1661-1671.

15. Goodwin TW. The Biochemistry of the Carotenoids. 2nd ed. London: Chapman and Hall; 1984.

16. Yuan JP, Chen F. Hydrolysis kinetics of astaxanthin esters and stability of astaxanthin of Haematococcus pluvialis during saponification. J Agric Food Chem. 1999;47:31-35.

17. Zhao L, Zhao G, Chen F, Wang Z, Wu J, Hu X. Different effects of microwave and ultrasound on the stability of (all-E)-astaxanthin. $J$ Agric Food Chem. 2006;54:8346-8351.

18. Hoffman TCM, Walsberg GE, DeNardo DF. Cloacal evaporation: an important and previously undescribed mechanism for avian thermoregulation. J Exp Biol. 2007;210:741-749.

19. McGraw KJ, Hill GE, Stradi R, Parker RS. The influence of carotenoid acquisition and utilization on the maintenance of speciestypical plumage pigmentation in male American goldfinches (Carduelis tristis) and northern cardinals (Cardinalis cardinalis). Physiol Biochem Zool. 2001;74:843-852.

20. Bauernfeind JC. Carotenoids as Colorants and Vitamin A Precursors: Technological and Nutritional Applications. New York: Academic Press; 1981.

21. Goldsmith TH, Butler BK. The roles of receptor noise and cone oil droplets in the photopic spectral sensitivity of the budgerigar, Melopsittacus undulatus. J Comp Pbysiol A. 2003;189:135-142.

22. Yuan JP, Chen F. Identification of astaxanthin isomers in Haematococcus lacustris by HPLC-photodiode array detection. Biotechnol Tech. 1997;11:455-459.

23. Stransky H, Schulze I. Carotenoids in Gallus-domesticus: comparative analysis of blood and retina of chickens and of egg-yolk. J Comp Physiol B. 1977;115:265-277.

24. Yamashita E, Maruyama Y, Katsuyama M, Tsushima M, Arai S, Matsuno T. The presence and origin of an apocarotenoid, galloxanthin in Ayu plecoglossus altivelis. Fisheries Sci. 1998;64:826-830.

25. Vecchi M, Glinz E, Meduna V, Schiedt K. HPLC separation and determination of astacene, semiastacene, astaxanthin, and other keto-carotenoids. J High Res Chromatogr. 1987;10:348-351.

26. Bjerkeng B, Hamre K, Hatlen B, Wathne E. Astaxanthin deposition in fillets of Atlantic salmon Salmo salar L. fed two dietary levels of astaxanthin in combination with three levels of alpha-tocopheryl acetate. Aquacult Res. 1999;30:637-646.

27. Østerlie M, Bjerkeng B, Liaaen-Jensen S. Plasma appearance and distribution of astaxanthin $\mathrm{E} / \mathrm{Z}$ and $\mathrm{R} / \mathrm{S}$ isomers in plasma lipoproteins of men after single dose administration of astaxanthin. $J$ Nutr Biochem. 2000;11:482-490.

28. Hart NS. Variations in cone photoreceptor abundance and the visual ecology of birds. J Comp Physiol A. 2001;187:685-697.

29. Lietz G, Henry CJK. A modified method to minimise losses of carotenoids and tocopherols during HPLC analysis of red palm oil. Food Chem. 1997;60:109-117.

30. Breithaupt DE. Enzymatic hydrolysis of carotenoid fatty acid esters of red pepper capsicum annum L. by a lipase from Candida rugosa (in German). Z Naturforsch C. 2000;55:971-975.

31. Wang Y, Connor SL, Wang W, Johnson EJ, Connor WE. The selective retention of lutein, meso-zeaxanthin and zeaxanthin in the retina of chicks fed a xanthophyll-free diet. Exp Eye Res. 2007;84:591-598.

32. Bernstein PS, Khachik F, Carvalho LS, Muir GJ, Zhao DY, Katz NB. Identification and quantitation of carotenoids and their metabolites in the tissues of the human eye. Exp Eye Res. 2001;72:215-223.

33. Schiedt K, Bischof S, Glinz E. Recent progress on carotenoid metabolism in animals. Pure Appl Chem. 1991;63:89-100.

34. Schiedt, K. New aspects of carotenoids metabolism in animals. In: Krinsky NI, ed. Carotenoids: Chemistry and Biology. New York: Plenum Press; 1990:262-266.

35. Wald G. Galloxanthin, a carotenoid from the chicken retina.J Gen Physiol. 1948;31:337-383. 